\title{
Quantitative Analysis of Alloy Structure by Aberration Corrected STEM
}

\author{
M.Weyland* and B. C. Muddle** \\ * Monash Centre for Electron Microscopy, Monash University, Bldg 81, VIC 3800, Australia \\ ** ARC Centre of Excellence for Design in Light Metals, Department of Materials Engineering, \\ Monash University, Vic 3800, Australia
}

The transmission electron microscope is invaluable in the study of metallic alloys, with the material properties often controlled by the structure and chemistry of nano-sized and nano-dispersed secondary phases. The mechanical properties of aluminium alloys in particular are often derived by the distribution of fine plate like precipitates, with well defined orientational relationships to the $\alpha-$ $\mathrm{Al}$ matrix. One interesting $\mathrm{Al}$ alloy system is the 'weldalite' $\mathrm{Al}-\mathrm{Cu}-\mathrm{Mg}-\mathrm{Li}-\mathrm{Ag}$ alloys, which are light, strong and also weldable. This makes them particularly useful in high end aerospace applications. While much is known about the microstructure of these alloys from classical TEM, there are many problems related to quantitative analysis of nano and atomic structure which remain uncertain. Two new techniques for the quantification of nanostructure, aberration corrected high angle annular dark field (HAADF) STEM and electron tomography, are here applied to one 'weldalite' alloy.

Aberration corrected HAADF STEM was carried out using an FEI Titan ${ }^{3} 80-300$. Images were acquired with a convergence angle of $15.1 \mathrm{mrad}$ and an inner collection angle of $42 \mathrm{mrad}$. An overview of the typical microstructure (Fig. 1a) shows, as expected from prior studies [1], two major families of plates. The majority are the $T_{I}$ phase which grow on the $\{111\}_{\alpha}$ planes. This phase, nominally $\mathrm{Al}_{2} \mathrm{CuLi}$, is also expected to accommodate both $\mathrm{Ag}$ and $\mathrm{Mg}$. The minor phase is tetragonal $\theta^{\prime}, \mathrm{Al}_{2} \mathrm{Cu}$ plates, on the $\{001\}_{\alpha}$ planes (Fig. 1b). Previous results have suggested that Li may segregate preferentially to the surface of $\theta^{\prime}$. And indeed the majority of the plates show a lower intensity at the plate surface (Fig. 1b); this is indicative of a low Z layer. Given the alloy composition, this is likely to be $\mathrm{Li}$ or $\mathrm{Mg}$. In addition a fine dispersion of other precipitates are also observed, in particular clustered around regions with large amounts of $\theta^{\prime}$. These have yet to have been fully identified but include $\mathrm{S}$ type (such as $S, S^{\prime}, S^{\prime \prime}$ ), shown in Fig. 1c, and $\Omega$, as well as well defined GPB zones [2] and a number of possible new phases.

Automated electron tomography was carried out using Xplore3D software on a FEI Tecnai F20 [3]. The tilt series data were aligned and reconstructed by routines coded in house using IDL. The high contrast reconstructions are ideal for semi-automatic three dimensional segmentation, allowing visualisation of the detailed morphology of the plates, Fig. 2, by surface rendering. This segmented data can then be used to give quantitative measurements of the microstructure; $T_{1}$ makes up $6.5 \%$ and $\theta^{\prime}$ just $0.35 \%$ of the volume. The errors in this rough measurement are considerable, mainly due to the inhomogeneous resolution in the reconstruction demonstrated by the gaps in some plates in Fig. 2 b). But the potential for measuring volume fractions, feature spacing, surface area and roughness from such reconstructions is considerable. What is required is improved reconstruction and segmentation that will minimise, and more importantly quantify, these errors. The 3D visualisation also allows unambiguous identification of 3D morphology such as the crossing between precipitates plates (Fig 2b). Both of these types of information are difficult, if not 
impossible, to resolve without significant uncertainty from conventional two dimensional micrographs.

\section{References}

[1] J.F. Nie and B.C. Muddle, Mat. Sci. and Eng. A319-321 (2001) 448

[2] Kovarik et al, Acta. Mat. 56 (2008) 4804

[3] Time on the Tecnai F20 at the Cornell University CCMR kindly provided by Prof. David Muller and support provided Mr John Grazul
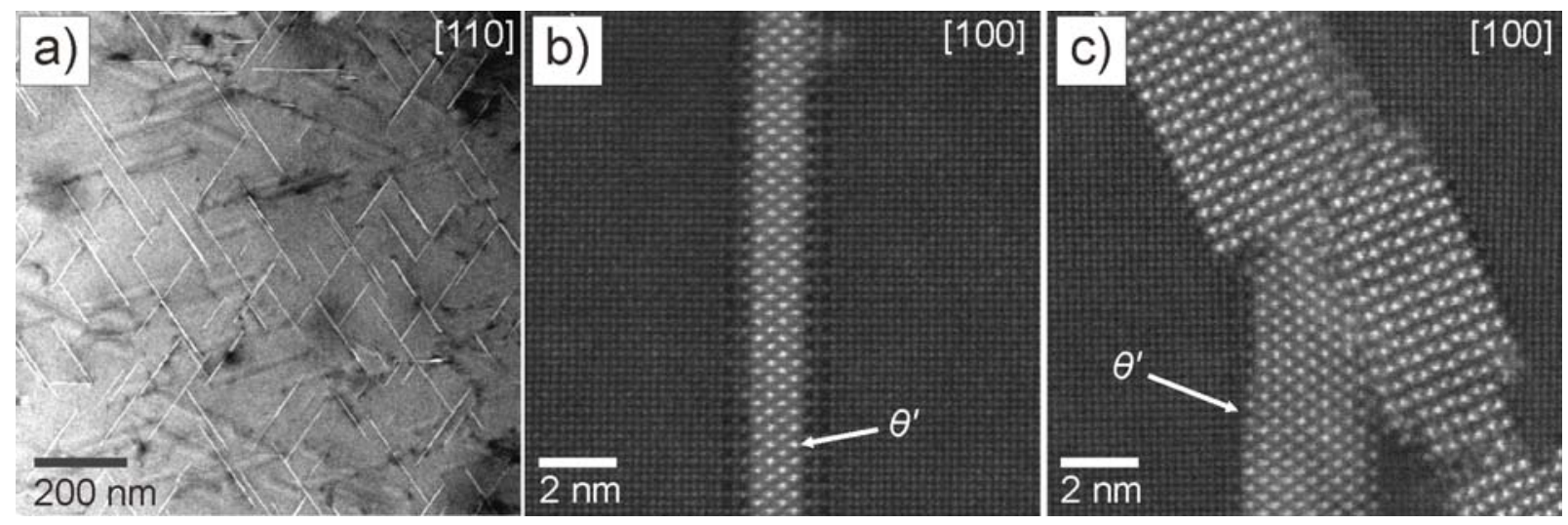

Fig. 1 a) HAADF STEM overview of the microstructure of the 'weldalite' alloy, showing mainly $T_{1}$ and a handful of $\theta^{\prime}$ plates. b) HR-STEM of a single $\theta^{\prime}$ plate, showing dark, possibly Li rich, capping layers. c) A single $\theta$ ' plate crossing a ' $S$ ' type plates on a [112] type plane.

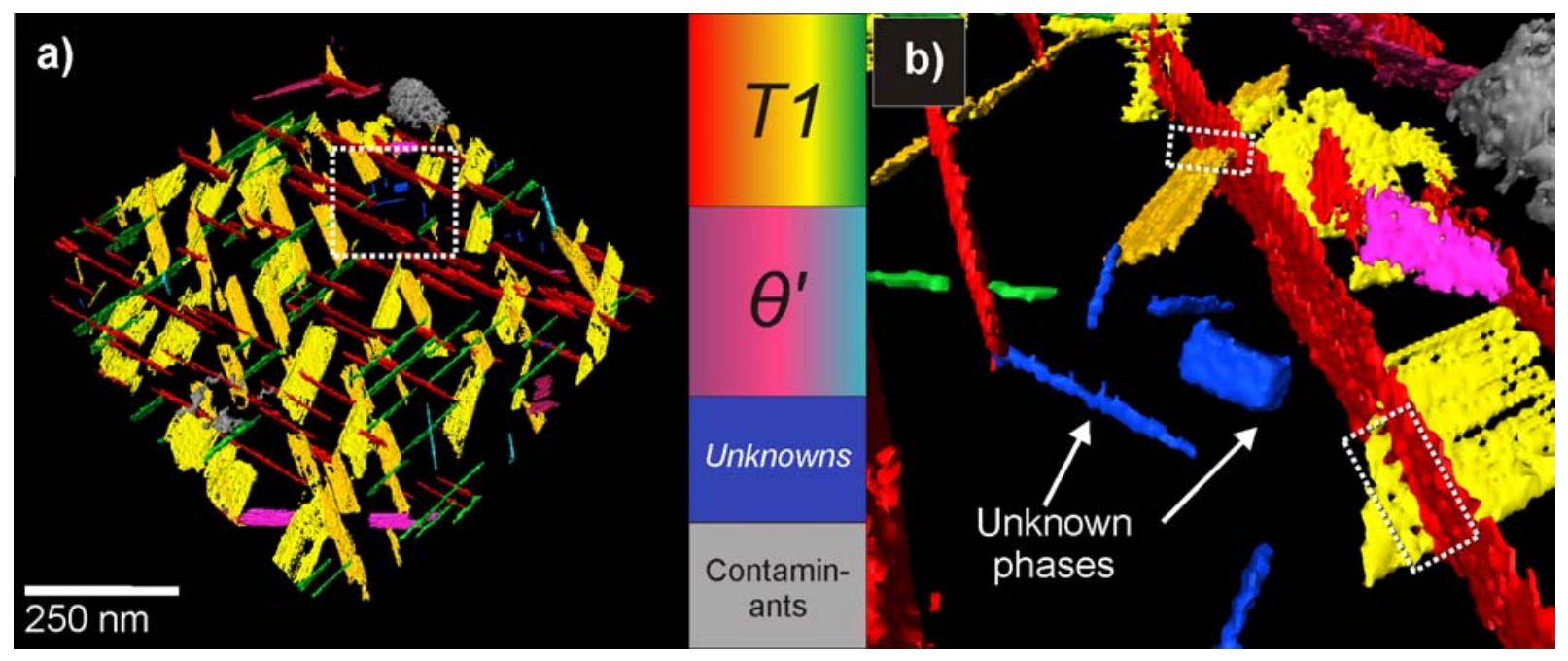

Fig. 2. Results of STEM HAADF electron tomography. a) Segmented surface visualisation of a tomographic reconstruction, key is shown in colour (grey) scale on right. b) Zoom in, and rotate, of boxed area in a) showing unknown phases (possible connected to T1 plates) and clear evidence of plate intersections (boxed areas). 